\title{
DYNAMIC AND SENSITIVITY ANALYSIS GENERAL NON-CONSERVATIVE ASYMMETRIC MECHANICAL SYSTEMS
}

\author{
ŽMINDÁK Milan ${ }^{1}$ \\ ${ }^{1}$ University of Žilina, Faculty of Mechanical Engineering, Department of Applied Mechanics, Univerzitná \\ 8215/1, 01026 Žilina, Slovak Republic, e-mail: milan.zmindak@fstroj.uniza.sk
}

\begin{abstract}
In this paper the concept of generalized form of proportional damping is proposed. Classical modal analysis of non-conservative continua is extended to multi DOF linear dynamic systems with asymmetric matrices. Mode orthogonality relationships have been generalized to non-conservative systems. Several discretization methods of continua are presented. Finally, an expression for derivatives of eigenvalues and eigenvectors of non-conservative system is presented. Examples are provided to illustrate the proposed methods.
\end{abstract}

KEYWORDS: non-conservative, asymmetric coefficient matrices, discretization, general damping, sensitivity

\section{Introduction}

Machine is a multi-parametric oscillating system. Its dynamic characteristics (properties) are dependent on design parameters and operational parameters. Design parameters define the shape, dimensions and material constants of all machine parts (components) and their mutual connection (linkage). Operational parameters characterise the mode of operation, for example revolutions, transmitted torque in rotary machines, state quantities of flowing medium in machines with flows, etc. In order to assess the dynamic behaviour of the machine, we require that the absolute value of displacement $u$ at any point $x$ in any operating mode and at all times $t$ does not exceed a certain conventional value $u_{\infty}$, i.e.

$$
u(x, y, z, t) \leq u_{\infty}
$$

In order to meet this simple criterion, we must first know the machine dynamic properties - dynamic analysis. In the second step we need to improve the dynamic properties that have proven unsatisfactory during the operation - dynamic synthesis. This includes the problems of tuning, reconstruction and optimisation [1-3]. Finally, it is necessary to verify whether the created mathematical model is a good approximation of the real system - identification.

In this paper we deal with modal analysis of asymmetric systems with real matrices $\mathbf{K}, \mathbf{C}$, M. Generally, these are non-conservative systems where it does not apply that the sum of potential and kinetic energy is constant. In the following sections we deal with methods of transformation (symmetrization) of these systems to a symmetric form. The objective of this subchapter is to explain some fundamental properties of non-classical structures and define the necessary and sufficient conditions for a non-classical system to become unconstrained, or that it can be solved in space $N$. The end of this chapter is dedicated to models of general damping and non-viscous damping. 


\section{Mathematical model of non-conservative continuum}

The objective of this part is to use methods of functional analysis $[4,5]$ to get to know and analyse spectral and modal properties of operators of mathematical models of nonconservative continuum. Using the above will allow us assessing the uniqueness (unambiguity) or ambiguity of orthonormal eigenfunctions that correspond to simple or multiple eigenvalues. Finally, we evaluate the consequences for numerical solution of a forced vibration.

We begin with a mathematical model of forced-oscillation non-conservative continuum described by a differential equation (or by a system of such equations) in the operator form

$$
M \ddot{\mathrm{v}}(\mathrm{t})+C \dot{\mathrm{v}}(\mathrm{t})+K \mathrm{v}(\mathrm{t})=f(t)
$$

This is a second order differential equation with respect to time $t$ with linear differential operators $K, C, M$ only with respect to the coordinates of the definition domain $\Omega$ with homogenous boundary conditions $L$ in $\left.(\Omega, t)\right|_{h}=0$, defining the state at the boundary $h$ of the definition domain $\Omega$ and with two initial conditions $\mathrm{v}_{0}, \dot{\mathrm{v}}_{0}$, defining the state in the beginning at time $t=0$. The system is acted upon by a force $f(t)$.

Since the differential operators $K, C, M$ are not a function of time $t$, equation, (1) can be expressed in the following form

$$
\begin{gathered}
{[K, \quad C, \quad M]\left[\begin{array}{c}
\mathrm{v}(t) \\
\dot{\mathrm{v}}(t) \\
\ddot{\mathrm{v}}(t)
\end{array}\right]=f(t)} \\
{\left[\begin{array}{lll}
K, & C, & M
\end{array}\right]\left[\begin{array}{c}
1 \\
T \\
T^{2}
\end{array}\right] \mathrm{v}(t)=f(t), T=d / d t}
\end{gathered}
$$

respectively in a short form as follows

$$
\begin{aligned}
\tilde{Z} \tilde{T} \mathrm{v}(t) & =f(t) \\
Z \mathrm{v}(t) & =f(t)
\end{aligned}
$$

\subsection{Modal analysis}

Modal analysis plays a central role in the vibrational studies of linear engineering structures. The methods for calculation eigenvalues was originally proposed for undamped structures whose inertia and stiffness properties can be represented by symmetric matrices or self-adjoint differential operators. In fact, practical experiences show that the damping cannot be neglected in many cases. For "classical damping" or "proportional damping", the modal analysis of undamped systems is applicable to damped systems. There are systems which inertia, stiffness and damping properties cannot be represented by symmetric matrices or selfadjoint differential operators. These kind of problems arise in the dynamics actively controlled structures and in many general non-conservative dynamic systems, for example, a moving vehicle on road, a missile on its trajectory, a ship's motion in seawater, etc.

A starting equation is the mathematical model (2). First, we investigate the spectral and modal properties of its operators, i.e. we solve the respective eigenvalue problem (EVP). From (2) we obtain equations of this problem for

$$
f=0, \mathrm{v}(t)=\mathrm{v} e^{s t}
$$


and that for both the function $\mathrm{v}$ and for the adjoined function $\mathrm{w}$ :

$$
\begin{aligned}
& \left(K+s C+s^{2} M\right) \mathrm{v}=0 \\
& \left(K+s C+s^{2} M\right)^{*} w=0
\end{aligned}
$$

The top right index “*” means the adjoint operator. As can be seen from (8), it is a quadratic eigenvalue problem in parameter $s$. Equation (2) is a second order equation with respect to $t$ and it is therefore useful to simplify this equation by adding a trivial identity

$$
M \dot{\mathrm{v}}(t)-M \dot{\mathrm{v}}(t)=0 x
$$

Then we obtain twice the number of first order equations with respect to $t$

$$
N \dot{u}(t)=P u(t)+g \gamma(t)
$$

where

$$
\begin{gathered}
N=\left[\begin{array}{cc}
C & M \\
M & 0
\end{array}\right], P=\left[\begin{array}{cc}
-K & 0 \\
0 & M
\end{array}\right] \\
u(\Omega, t)=\left[\begin{array}{c}
\mathrm{v}(t) \\
\dot{\mathrm{v}}(t)
\end{array}\right], g=\left[\begin{array}{c}
f \\
0
\end{array}\right] .
\end{gathered}
$$

To equation (11) then belongs the eigenvalue problem (EVP)

$$
(P-s N) u=0=(p-s N)^{*} u^{*}
$$

with a nontrivial solution of $s_{v}, u_{v}, u_{v}^{*}$ satisfying the EVP equations

$$
\left(P-s_{v} N\right) u_{v}=0=\left(p-s_{\sigma} N\right)^{*} u_{\sigma}^{*}
$$

After assigning the scalar product to the first equation by function $u_{\sigma}^{*}$ and by the second function $u_{v}$ we obtain:

$$
\begin{aligned}
&\left(u_{\sigma}^{*},\left\langle P-s_{v} N\right\rangle u_{v}\right)=0,\left(u_{v},\left\langle P-s_{\sigma} N\right\rangle^{*} u_{\sigma}^{*}\right)=0 \\
&\left(\left\langle P-s_{\sigma} N\right\rangle u_{v}, u_{\sigma}^{*}\right)=0 \\
&\left(u_{\sigma}^{*},\left\langle P-s_{\sigma} N\right\rangle u_{v}\right)=0
\end{aligned}
$$

from which we obtain

$$
\begin{aligned}
& \left(u_{\sigma}^{*}, N u_{v}\right) s_{\sigma}=\left(u_{\sigma}^{*}, P u_{v}\right)=\left(u_{\sigma}^{*}, N u_{v}\right) s_{v} \\
& \left(s_{\sigma}-s_{v}\right)\left(u_{\sigma}^{*}, N u_{v}\right)=0
\end{aligned}
$$

This leads to general formulas

$$
\left(u_{\sigma}^{*}, N u_{v}\right)=\delta_{\sigma v}, \quad\left(u_{\sigma}^{*}, P u_{v}\right)=s_{v} \delta_{\sigma v}
$$

If the eigenvalue $s_{v}$ is $n_{v}$-fold, then it is attributed linear independent eigenfunctions $u_{j v}, u_{j v}^{*}$ in the number $n_{v}$. So we assume that these are continuous of a simple structure [6]. From these eigenvalues, we create scalar spectral sub-matrices $S_{v}=s_{v} I_{v}$ of the $n_{v}$-th order, and the respective modal sub-matrices $U_{v}=\left[u_{j v}\right], W_{v}^{*}=\left[w_{j v}^{*}\right]$ that satisfy the EVP equations 


$$
P U_{v}-N U_{v} S_{v}=0=P^{*} U_{v}^{*}-N^{*} U_{v}^{*}-N^{*} U_{v}^{*} \bar{S}_{v}^{1}
$$

${ }^{1}$ As the operators $\mathrm{K}, \mathrm{C}, \mathrm{M}$, and hence also $\mathrm{P}, \mathrm{N}$ are real, in addition to submatrices $S_{v}, U_{v}, U_{v}^{*} x$ a solution is also in complex conjugate sub-matrices $\bar{S}_{v}, \bar{U}_{v}, \bar{U}_{v}^{*}$.

and meet the conditions of orthogonality and norm

$$
\left(U_{v}^{*}, N U_{v}\right)=I_{v},\left(U_{v}^{*}, P U_{v}\right)=S_{v}
$$

Solution to equation (11) is then searched in the following form

$$
u(t)=\sum_{v} U_{v} c_{v}(t)
$$

Substituting to (11), assigning scalar products to the sub-matrix $U_{\sigma}^{*}$ and with respect to (20), by the method of variation of constants we obtain the solution

$$
\begin{aligned}
& \sum_{v} N U_{v} \dot{c}_{v}(t)=\sum_{v} P U_{v} c_{c}(t)+g \gamma(t) \\
& \sum_{v}\left(U_{\sigma}^{*}, N U\right) \dot{c}_{v}(t)=\sum_{v}\left(U_{\sigma}^{*}, P U_{v}\right) c_{v}(t)+\left(U_{\sigma}^{*}, g\right) \gamma(t) \\
& c_{v}(t)=S_{v} c_{v}(t)+\left(U_{v}^{*}, g\right) \gamma(t) \\
& c_{v}(t)=e^{t S_{v}} a_{v}(t) \\
& \quad S_{v} e^{t S_{v}} a_{v}(t)+e^{t S_{v}} \dot{a}_{v}(t)=S_{v} e^{t S_{v}} a_{v}(t)+\left(U_{v}^{*}, g\right) \gamma(t) \\
& \dot{a}_{v}(t)=e^{-t S_{v}} v \gamma(t)+\left(U_{v}^{*}, g\right) \\
& a_{v}(t)=a_{o v}+\int_{0}^{t} e^{-\tau S_{v}} \gamma(\tau) d \tau\left(U_{v}^{*}, g\right)
\end{aligned}
$$

\subsection{Forced vibration}

From the general solution (22) we derive formulas for forced oscillation in the transient mode (TM), respectively in the harmonic mode (HM)

TM:

$$
u(t)=\sum_{v} U_{v} e^{t S_{v}} a_{o v}+\sum_{v} U_{v} \Phi_{v}(t)\left(U_{v}^{*}, g\right)
$$

where

$$
\begin{array}{ll}
\Phi_{v}(t)=\phi_{v}(t) I_{v}, & \phi_{v}(t)=\int_{0}^{t} e^{(t-\tau) s_{v}} \gamma(\tau) d \tau \\
a_{o v}=\left(U_{v}^{*}, N u_{o}\right), & u_{0}(t)=\left[\begin{array}{c}
\mathrm{v}_{o} \\
\dot{\mathrm{v}}_{o}
\end{array}\right]
\end{array}
$$

HM:

$$
u(t)=\sum_{v} U_{v} e^{t s_{v}} c_{0 v}+\sum_{v}\left(i \omega I_{v}-S_{v}\right)^{-1}\left(U_{v}^{*}, g\right) e^{i \omega t}
$$

where

$$
c_{o v}=a_{o v}-\left(i \omega I_{v}-S_{v}\right)^{-1}\left(U_{v}^{*}, g\right)
$$




$$
\begin{gathered}
\Phi_{v}(t)=\varphi_{v}(t) I_{v} \\
\phi_{v}(t)=\int_{0}^{t} e^{(t-\tau) S_{v}} \gamma(\tau) e^{i \omega t} d \tau=\left(i \omega-S_{v}\right)^{-1}\left(e^{i \omega t}-e^{s_{v} t}\right)
\end{gathered}
$$

From formulas (24) and (25) we obtain the forced oscillation formulas

TM:

$$
\begin{aligned}
& \mathrm{v}(t)=\sum_{v} V_{v} e^{t S_{v}} a_{0 v}+\sum_{v} V_{v} \Phi_{v}(t)\left(W_{v}, f\right) \\
& \dot{\mathrm{v}}(t)=\sum_{v} V_{v} S_{v} e^{t S_{v}} a_{0 v}+\sum_{v} V_{v} S_{v} \Phi_{v}(t)\left(W_{v}, f\right)
\end{aligned}
$$

HM:

$$
\begin{aligned}
& \mathrm{v}(t)=\sum_{v} V_{v} e^{t S_{v}} c_{0 v}+\sum_{v} V_{v}\left(i \omega I_{v}-S_{v}\right)^{-1}\left(W_{v}, f\right) e^{i \omega t} \\
& \dot{\mathrm{v}}(t)=\sum_{v} V_{v} S_{v} e^{t S_{v}} c_{0 v}+\sum_{v} V_{v} S_{v}\left(i \omega I_{v}-S_{v}\right)^{-1}\left(W_{v}, f\right) e^{i \omega t}
\end{aligned}
$$

Let us note the formulas for velocity in formulas (29). If we derive them by differentiating the formula for forced displacement, we obtain

$$
\begin{gathered}
\dot{\mathrm{v}}(t)=\partial \mathrm{v}(t) / \partial t=\sum_{v} V_{v} S_{v} e^{t S_{v}} a_{o v}+\sum_{v} V_{v} S_{v} \Phi(t)\left(W_{v}, f\right) e^{i \omega t}+\sum_{v} V_{v}\left(W_{v}, f\right) \gamma(t) \\
V_{v}\left(W_{v}, f\right)=0
\end{gathered}
$$

This consideration gives rise to general formulas for forced oscillation, which we breakdown for displacement, velocities in the transition mode (TM) and in the harmonic mode (HM), and the steady-state harmonic mode (SSHM), when the influence of the initial conditions disappears

TM:

$$
\begin{aligned}
& \mathrm{v}(t)=\sum_{v} V_{v} e^{t S_{v}} a_{0 v}+\sum_{v} V_{v} \Phi_{v}(t)\left(W_{v}, f\right) \\
& \dot{\mathrm{v}}(t)=\sum_{v} V_{v} S_{v} e^{t S_{v}} a_{0 v}+\sum_{v} V_{v} S_{v} \Phi_{v}(t)\left(W_{v}, f\right)
\end{aligned}
$$

where

$$
\begin{aligned}
& a_{0 v}=\left(W, M \dot{\mathrm{v}}_{0}+C \mathrm{v}_{0}+M \mathrm{v}_{0} S_{v}\right) \\
& \Phi_{v}(t)=\phi_{v}(t) I_{v}, \quad \phi_{v}(t)=\int_{0}^{t} e^{s_{v}(t-\tau)} \gamma(\tau) d \tau \\
& e^{t S_{v}}=e^{s_{v} t} I_{v}
\end{aligned}
$$

HM:

$$
\begin{aligned}
& \mathrm{v}(t)=\sum_{v} V_{v} e^{t S_{v}} c_{0 v}+\sum_{v} V_{v}\left(i \omega I_{v}-S_{v}\right)^{-1}\left(W_{v}, f\right) e^{i \omega t} \\
& \dot{\mathrm{v}}(t)=\sum_{v} V_{v} S_{v} e^{t S_{v}} c_{0 v}+\sum_{v} V_{v} S_{v}\left(i \omega I_{v}-S_{v}\right)^{-1}\left(W_{v}, f\right) e^{i \omega t} \\
& c_{0 v}=a_{0 v}-\left(i \omega I_{v}-S_{v}\right)^{-1}\left(W_{v}, f\right)
\end{aligned}
$$

SSHM: 


$$
\begin{aligned}
& \mathrm{v}(t)=\sum_{v} V_{v}\left(i \omega I_{v}-S_{v}\right)^{-1}\left(W_{v}, f\right) e^{i \omega t} \\
& \dot{\mathrm{v}}(t)=\sum_{v} V_{v} S_{v}\left(i \omega I_{v}-S_{v}\right)^{-1}\left(W_{v}, f\right) e^{i \omega t}
\end{aligned}
$$

Note that when we enter $\omega=0$ in the first formula (36), we obtain the formula for static displacement $\mathrm{v}_{s}$, due to static load $f$ as a limiting case of the dynamic phenomenon

$$
\begin{aligned}
& \mathrm{v}(t)=\sum_{v} V_{v}\left(i \omega I_{v}-S_{v}\right)^{-1}\left(W_{v}, f\right) e^{i \omega t} \\
& \dot{\mathrm{v}}(t)=\sum_{v} V_{v} S_{v}\left(i \omega I_{v}-S_{v}\right)^{-1}\left(W_{v}, f\right) e^{i \omega t}
\end{aligned}
$$

\section{Discretization of the continuum}

Continuum mechanics is based on a system of partial differential equations (PDE) with appropriate initial and boundary conditions. The analytical solution of PDE can be obtained only if the shape of the considered body is sufficient simple, e.g., if it is rotationally symmetric, quadrilateral, infinite, etc. Successful solving of complex engineering problems begins with defining a physical model. The physical model is then transformed to a mathematical model. To formulate the equations of motion we make use of techniques employed in theory on mechanics of deformable bodies which leads to a set of three equations of motion, in terms of the displacements $u(x, y, z, t), \mathrm{v}(x, y, z, t), \mathrm{w}(x, y, z, t)$, which must be satisfied at every point of the system and which are subjected to initial conditions and at the boundaries of the system. Each of the variables $x, y$, and $z$ can take an infinity of values within the region occupied by the system, so the system poses an infinite of degrees of freedom.

The classification of systems as discrete or continuous is quite often arbitrary. The same system can be regarded at times as discrete and described by ordinary differential equation and other times as continuous and described partial differential equations.

A solution to the mathematical model is usually obtained by numerical methods that are called approximation. Finite element analysis is now abundantly performed in various branches of engineering design and scientific research. A number of commercial computer programs are employed for solution many industrial problems, including modelling of technological processes $[7,8]$.

The transformation process of a continuous mathematical model to a model with a finite number of degrees of freedom is called discretization. In statics we deal with discretization in space. In dynamics it is discretization in space and time. We mainly use the following discretization methods [9]:

- Finite element method (FEM)

- Differential method

- Boundary element method (BEM)

- $\quad$ Finite volume method (FVM)

- Spectral methods

- Meshless methods 
The area of the continuum computational mechanics is currently divided into two leading methods. While the mechanics of bodies (stress analysis, and dynamics) is absolutely dominated by finite elements, fluid dynamics [10] is dominated to a certain extent by the finite volume method (FVM). Both the methods are based on different analytical principles:

1. FEM is predominantly based on the variational principle; it uses predefined shape functions for the element topology. It is easily extended to a higher order discretization. It leads to sparse matrices, usually with good conditionality, and solving the resulting system is carried out using direct or iterative solvers.

2. FVM, on the other hand, usually shows a second order accuracy. It is based on an integral form of basic equations; it uses segregated solvers, where constraint conditions and nonlinearities are solved iteratively. The method leads to diagonally dominant matrices suitable for iterative solvers. The main applications of this method are in computational fluid dynamics (CFD) and in fluid-structure interaction (FSI) [11]. Here, following discretization, we obtain hundreds of millions of equations. Their solving produces a sufficiently accurate solution to such problems as, for example, aerodynamics of vehicles or aircraft, internal combustion in engines, nuclear reactor models, etc.

In the following sections we briefly mention the basic principles of FEM. FEM formulation in displacements is the basis of most commercial software programs, such as ABAQUS, ADINA, ANSYS, etc.

According to the variational principle used, FEM is classified into three main groups [12]:

1. Rayleigh-Ritz formulation.

2. Galerkin formulation.

3. Least-squares formulation.

\subsection{Rayleigh-Ritz method}

The Rayleigh-Ritz method looks for a minimum total potential energy and thus the numerical solution based on this principle has the characteristics of the best approximation, i.e. the difference between the FEM solution and the exact solution is minimised with respect to a certain energy standard [13]. Moreover, this FEM formulation leads (at least in linear, but often even in nonlinear problems) to a symmetric positive definite system of linear algebraic equations. This formulation is mainly used in the mechanics of bodies and in heat conduction. Note that most of the current commercial FEM software programs are based on this method.

\subsection{Galerkin method}

The Galerkin method is based on the formulation using weighted residuals. The unknown function is approximated using unknown parameters and test (basis) functions. Algebraic functions that allow solving the problem numerically are formulated by "weighted residuals" while using appropriately chosen "test functions". For equations with a self-adjoint and positive definite operator, the Galerkin formulation leads to the same system of equations as the Rayleigh-Ritz formulation.

Note that the Galerkin method is more general, since it can be applied even to non-selfadjoint equations, such as for fluid flow, where the convection component (flow) dominates over the conduction component. Here, the Galerkin method does not manifest the best approximation feature. Convection components are of the first order and, therefore, non-selfadjoint. In practice, solving of flow leads to significant oscillations, which are removed by the 
mesh smoothening, and to stabilisation numerical procedures, which then leads to solving very large systems of equations and to the need for supercomputers to solve them.

\subsection{FEM based on the least squares method}

Formulation of finite elements by least squares is based on minimising the residual (error) in terms of least squares [14]. In contrast to the Galerkin formulation, which is only conceptual and without practical significance and, therefore, in most cases requires further mathematical manipulations for the realistic calculation of the practical problem, least squares are the final computational expression for any problem. Its disadvantage is that it can be applied only to first order differential equation, and hence higher-order differential equations need to be transformed to first order equations by introducing other variables, thus increasing the number of degrees of freedom for the nodal point.

\subsection{FEM based on reciprocity}

Formulation of finite elements using the reciprocity principle is based on the Trefftz functions or fundamental solution for the same continuum (body), or subdomain (element). Meanwhile, each auxiliary state can be linear even for nonlinear problems. The disadvantage is an asymmetric stiffness matrix even for linear problems. Linear problems lead to integration only along the surface of the domain, in our case the element. The method is under development and its advantages are being studied. Given the basis of formulation, we can name this method a multi-domain formulation of boundary elements, because both are based on the same equations. Closely related to this formulation is the hybrid FEM formulation, and in particular the hybrid strain formulation. However, we are not dealing with formulation in this part, because only its applications to solving linear problems have been known so far. The reason why this method has not been used so far for nonlinear problems is that it requires direct application of Trefftz functions to formulate the problem, and Trefftz functions for nonlinear tasks have not been used yet. Such Trefftz functions can be defined for a given configuration (in nonlinear problems - it must be the instantaneous configuration of the body), for example in terms of least squares through a discrete set of points of the relevant element (subdomains) [15].

\subsection{Boundary element Method}

The basis of any boundary integral equation (BIE) in the classical theory of elasticity is Betti's theorem on reciprocity (1872) and the application of elastic potentials to satisfy equilibrium equations using Somigliana's identity (1885). In recent years, many BIE formulations have been made using the weighted residuals method (WRM). Note that WRM is simply applied and is easier to understand, especially for those who know the basics of FEM. On the other hand, WRM obscures many fundamental strengths and weaknesses of BIE. Therefore we prefer using the classic approach $[16,17]$ to derive basic integral equations.

A standard FEM is derived in displacements, which means that the only unknowns are displacements. These displacements are approximated using the functions that have satisfied the prescribed (imposed) boundary conditions in displacements. Stress and load are secondary unknowns that are calculated after calculating displacements. The final result is lower precision of calculated stress and load. On the other hand, the boundary element method (BEM) is a mixed formulation where the unknowns are both displacements and loads. Therefore we also approximate the load and the approximation functions are selected so that they satisfy also the boundary conditions in loads. This is why loads obtained by BEM are usually more accurate than those in FEM.

Another difference between the methods is in the selection of weighting functions. A classic FEM is the Bubnov-Galerkin method in which weighting functions are selected as the 
fundamental solution of the governing differential equation. This leads to elimination of the integral over the investigated domain by Dirac delta (impulse) function displacement, resulting in a boundary formulation for the unknown field of displacement and load.

\section{$4 \quad$ Asymmetric damped discrete systems}

Analysis of damped, i.e. non-conservative, systems is much more complex than that of conservative systems [18]. Although solving equation of motion, or studying the mathematical model, is based on linear algebra methods, yet a rather complex mathematical apparatus is used, and it is only suitable for examining the system properties. From a mathematical point of view, it is examining the linear dynamic systems of general, i.e. Jordanian, structure with dominant occurrence of multiple eigenvalues [19]. Equation of motion in a discrete form and with $n$ degrees of freedom has an analogous form as equation (2)

$$
\mathbf{M} \ddot{\mathbf{q}}+\mathbf{C} \dot{\mathbf{q}}+\mathbf{K q}=\mathbf{f}(t)
$$

where $\mathbf{M}$ is the mass matrix, $\mathbf{C}$ is the damping matrix, and $\mathbf{K}$ is the stiffness matrix of $N x N$ degree. General displacement $\mathbf{q}(t)$ and excitation $\mathbf{f}(t)$ are $N$-dimensional vectors. It is assumed for classic systems that $\mathbf{M}$ is symmetric and positive definite, and $\mathbf{C}$ and $\mathbf{K}$ are symmetric and positive-semidefinite.

The use of modern control devices leads to asymmetric matrices that also loose their positive definiteness. Asymmetry of $\mathbf{C}$ and $\mathbf{K}$ is usually associated with gyroscopic forces. Linear systems with asymmetric matrices $\mathbf{M}, \mathbf{C}$ and $\mathbf{K}$, which are not positive definite, are called non-classic systems. These systems are mainly found in microdynamics. As long as matrices $\mathbf{M}, \mathbf{C}$ and $\mathbf{K}$ can be transposed to a symmetric shape, we talk about symmetrization of non-classic systems [20]. Note that for mechatronic systems, the system of differential equations (38) is supplemented by systems of algebraic equations. With some exceptions, analytical solving of the above systems is not possible and we must therefore use numerical methods. However, equation of motion (38) can be also transformed to the state space. This is more convenient for problems relating to controlled movement.

\subsection{Solving in $2 \mathrm{~N}$-dimensional space}

In this case, solving the system (38) is transformed to state space $2 \mathrm{~N}$. By adding identity $\mathbf{M} \dot{\mathbf{q}}-\mathbf{M} \dot{\mathbf{q}}=\mathbf{0}$ to system we obtain a system of two matrix equations

$$
\begin{aligned}
& \mathbf{M} \ddot{\mathbf{q}}+\mathbf{C} \dot{\mathbf{q}}+\mathbf{K q}=\mathbf{f}(t) \\
& \mathbf{M} \dot{\mathbf{q}}-\mathbf{M} \dot{\mathbf{q}}=\mathbf{0}
\end{aligned}
$$

Then we obtain in the compact form

$$
\mathbf{A} \dot{\mathbf{x}}(t)+\mathbf{B} \mathbf{x}(t)=\mathbf{p}(t)
$$

where

$$
\mathbf{A}=\left[\begin{array}{cc}
\mathbf{C} & \mathbf{M} \\
\mathbf{M} & \mathbf{0}
\end{array}\right], \mathbf{B}=\left[\begin{array}{cc}
\mathbf{K} & \mathbf{0} \\
\mathbf{0} & -\mathbf{M}
\end{array}\right], \mathbf{x}(t)=\left[\begin{array}{c}
\mathbf{q}(t) \\
\dot{\mathbf{q}}(t)
\end{array}\right], p(t)=\left[\begin{array}{c}
\mathbf{q}(t) \\
\dot{\mathbf{q}}(t)
\end{array}\right]
$$

Since matrices $\mathbf{A}$ and $\mathbf{B}$ are asymmetric for calculation of eigenvalues (and eigenvectors), we must consider the eigenproblem from the right and from the left

$$
(s \mathbf{A}+\mathbf{B}) \mathbf{z}=\mathbf{0}, \mathbf{y}^{H}(s \mathbf{A}+\mathbf{B})=\mathbf{0}
$$


where $s$ is the eigenvalue, $\mathbf{z}$ the right-hand eigenvector, and $\mathbf{y}$ is the left-hand eigenvector. The top left index " $H$ " denotes the Hermitian conjugate vector $\mathbf{y}^{H}=(\overline{\mathbf{y}})^{T}$ and "'- " is the complex conjugate number. Let us note that vectors $\mathbf{z}_{j}$ and $\mathbf{y}_{j}$ are orthogonal and can be normalised so as to satisfy also the conditions of orthonormality [19].

\subsection{Solving in $\mathbf{N}$-dimensional space}

Solving equation of motion (38) in $\mathrm{N}$-dimensional space means to investigate the spectral and modal properties of the system and its response to the known curve of loading force without transforming the original problem to $2 \mathrm{~N}$-space (state space). The system response is then simply the sum of the homogenous solution (eigen oscillation) and the particular solution which represents forced (driven) oscillations should eigen oscillation disappear. It is important to note that solving the equation of motion in space $\mathrm{N}$ has the advantage that matrices $\mathbf{M}, \mathbf{C}, \mathbf{K}$ are of the $\mathrm{N}$ degree, while in $2 \mathrm{~N}$-space we work with $2 \mathrm{~N}$-degree matrices $\mathbf{A}$ and $\mathbf{B}$.

In general, matrices $\mathbf{M}, \mathbf{C}, \mathbf{K}$ are asymmetric. Therefore, the eigenvalues are not real but complex. The eigenvalue problem becomes a quadratic problem. Assuming a solution in the form $\mathbf{v}(t)=\mathbf{v} e^{s t}$, then after substituting to (38) we obtain

$$
\left(\mathbf{M} s^{2}+\mathbf{C s}+\mathbf{K}\right) \mathbf{u}=\mathbf{0}
$$

Given the matrices $\mathbf{M}, \mathbf{C}, \mathbf{K}$ asymmetry, it is necessary to consider also the adjoint eigenvalue problem (problem from the left)

$$
{ }^{H} \mathbf{w}\left(\mathbf{M} s^{2}+\mathbf{C s}+\mathbf{K}\right)={ }^{T} \mathbf{0}
$$

where ${ }^{H} \mathbf{w}$ is the Hermitian conjugate vector to vector $\mathbf{w}\left({ }^{H} \mathbf{w}=\overline{\mathbf{w}}^{T}\right)$. Equation (41) has the form

$$
\left(\mathbf{M}^{T} s^{2}+\mathbf{C}^{T} s+\mathbf{K}^{T}\right) \mathbf{w}=\mathbf{0}
$$

Based on the Frobénius theorem for obtaining a nontrivial solution, the following applies

$$
\operatorname{det}\left(\mathbf{M} s^{2}+\mathbf{C s}+\mathbf{K}\right)=0
$$

Equation (44) is a polynomial equation of the $2 \mathrm{~N}$ degree, and its solving provides us $2 \mathrm{~N}$ eigenvalues that occur in complex conjugate pairs. For different eigenvalues, orthonormality conditions are satisfied in the form [22]

$$
\left.\begin{array}{c}
{ }^{H} \mathbf{w}_{\mathrm{j}}\left[\left(s_{i}+s_{j}\right) \mathbf{M} \mathbf{v}_{i}+\mathbf{C}\right] \mathbf{v}_{i}=0 \\
{ }^{H} \mathbf{w}_{j}\left(2 s_{j} \mathbf{M}+\mathbf{C}\right) \mathbf{v}_{j}=1
\end{array}\right\}
$$

As regards the calculation of eigenvectors of asymmetric non-conservative systems, these cannot be calculated by simple procedures. One of the possibilities is to determine these vectors by the left-hand-side and right-hand-side vectors of the corresponding asymmetric conservative system.

\section{Example 1 - three degrees of freedom}

To illustrate the calculation of left-hand-side and right-hand-side eigenvectors and eigenvalues, we present the following example with three degrees of freedom. Matrices $\mathbf{M}, \mathbf{C}, \mathbf{K}$ are defined as follows: 


$$
\begin{gathered}
\mathbf{M}=\left[\begin{array}{ccc}
0.5574 & 1.3858 & 1.3858 \\
0.7070 & 0.7070 & -0.7070 \\
0.4620 & -1.1914 & -0.1914
\end{array}\right], \\
\mathbf{C}=\left[\begin{array}{ccc}
2.6710 & 2.9592 & 2.9651 \\
0.4843 & 1.2606 & -0.6119 \\
0.2875 & -0.5808 & 1.2272
\end{array}\right], \mathbf{K}=\left[\begin{array}{ccc}
1.3748 & 10.9440 & 25.2975 \\
1.2625 & 2.8770 & -17.4195 \\
0.7455 & -4.1244 & 0.8625
\end{array}\right]
\end{gathered}
$$

As can be seen, all the three matrices are asymmetric, and it is easy to show that they are not positive definite. By solving the general problem of eigenvalue without damping, we obtain eigenfrequencies $\omega_{1}=1.3506, \omega_{2}=3.0913, \omega_{3}=4.8527$ and the matrix of left-hand-side and right-hand-side vectors

$$
\begin{gathered}
\mathbf{U}=\left[\mathbf{u}_{1}, \mathbf{u}_{2}, \mathbf{u}_{3}\right]=\left[\begin{array}{ccc}
1.0741 & -0.6240 & 0.4421 \\
-0.0292 & 0.9635 & -0.5428 \\
-0.0047 & -0.00290 & 0.8731
\end{array}\right] \\
\mathbf{V}=\left[\mathbf{v}_{1}, \mathbf{v}_{2}, \mathbf{v}_{3}\right]=\left[\begin{array}{ccc}
0.3082 & 0.4876 & 0.5167 \\
0.5025 & 0.3000 & -0.7973 \\
0.8983 & -0.9954 & 0.5915
\end{array}\right]
\end{gathered}
$$

If we consider damping, then the eigenvalues are $s_{1}=-0.7725+1.1965 \mathrm{i}$, $\mathrm{s}_{2}=-0.7251+3.056 \mathrm{i}, \mathrm{s}_{3}=-1.3949+4.0392 \mathrm{i}$ and also their corresponding complex conjugate values. Complex eigenfrequencies are obtained from relation $\lambda_{i}=s_{i} / i$

$$
\begin{array}{r}
\mathbf{U}_{d}=\left[\begin{array}{ccc}
1.0162+0,0819 i & -0.7395+0.1175 i & 0.6187+0.5750 i \\
0.0665-0,1357 i & 0.9530-0.0994 i & -0.7821-0.2688 i \\
0.0310-0.0527 i & -0.0063+0.1582 i & 0.8802+0.0060 i
\end{array}\right] \\
\mathbf{W}=\left[\begin{array}{ccc}
0.3426-0.0505 i & 0.5248+0.2880 i & 0.5117-0.0358 i \\
0.4676+0,0709 i & 0.0162+0,0713 i & -0.7731+0.0691 i \\
0.9109-0.0475 i & -1.0789+0.6182 i & 0.7347+0.5180 i
\end{array}\right]
\end{array}
$$

\subsection{Asymmetric systems with general proportional damping}

Damping is a complex physical phenomenon and its consideration most often utilises the so-called Rayleigh damping. In this case we express the damping matrix as

$$
\mathbf{C}=\alpha \mathbf{M}+\beta \mathbf{K}
$$

where $\alpha$ and $\beta$ are real values (Rayleigh coefficients). This type of damping is considered internal damping. Its major drawback is that damping is applied across the board for all material points of the body that have a non-zero velocity. Since the model does not distinguish between rigid motion and deformation, its use is limited to cases where a body's rigid motion relative to deformation is negligible.

If we want to use the modal method also for damped systems, the simplest way is to assume proportional damping. Using modal transformation $\mathbf{u}=\mathbf{V} \mathbf{q}$ we obtain from (37), for zero damping, an overdetermined system of equations for the unknown coefficients $\alpha$ and $\beta$ 


$$
2 \varsigma_{i} \Omega_{i}=\alpha+\beta \Omega_{i}^{2}, \quad i=1, \ldots, n
$$

where $\varsigma_{i}(I=1, n)$ is the damping coefficient (factor). Note that for a system with two degrees of freedom the system (48) solution is unambiguous. For systems with many degrees of freedom, that is mainly for systems discretised using the finite element method (FEM), we take into account the fact that the resulting dynamic response is contributed to by only the first few eigenmodes, $\mathrm{m}$ « $\mathrm{n}$. The effect of higher eigenmodes is negligible. If we express from (48) the damping coefficient $\varsigma_{i}$, we obtain

$$
\varsigma=\frac{\alpha}{2 \Omega_{i}}+\frac{\beta \Omega_{i}^{2}}{2}, i=1, \ldots, n
$$

From (48) we see that the member containing $\beta / 2$ is dominant for sufficiently stiff structures. So it can be assumed that the damping coefficient is a linear proportional function of frequency. Based on the above, the damping coefficients for $1<i<m$ are calculated as follows

$$
\varsigma_{i}=\frac{\varsigma_{m}-\varsigma_{1}}{\Omega_{m}-\Omega_{1}}\left(\Omega_{i}-\Omega_{1}\right)+\zeta_{1} \text { for } i \leq m
$$

For eigenfrequencies larger than $m$, the damping coefficients values are extrapolated as

$$
\varsigma_{i}=\frac{\varsigma_{m}-\varsigma_{1}}{\Omega_{m}-\Omega_{1}}\left(\Omega_{m+i}-\Omega_{m 1}\right)+\zeta_{1} \text { for } m<i \leq 2.5 m,
$$

while we are considering $2.5 \mathrm{~m}$ eigenmodes. Furthermore, we calculate coefficients $\alpha$ and $\beta$ for the values $\zeta_{1}, \zeta_{m}, \Omega_{1}, \Omega_{m}$ and $\zeta_{1}, \zeta_{2,5 m}, \Omega_{1}, \Omega_{2.5 m}$. The resulting values of $\alpha$ and $\beta$ are obtained as average of these values. In the following sections we present more general conditions that must be satisfied in order for a damped system to have classic eigenmodes.

If we consider proportional damping, then system (38) has classic eigenmodes. It can be proven that a linear damped system can have classic eigenmodes also for the so-called general damping [23]. This first the so-called commutative damping when the system matrices satisfy the following relations:

$$
\text { 1) } \mathbf{K M}^{-1} \mathbf{C}=\mathbf{C M}^{-1} \mathbf{K} \text {, 2) } \mathbf{M K}^{-1} \mathbf{C}=\mathbf{C K}^{-1} \mathbf{M} \text {, 3) } \mathbf{M C}^{-1} \mathbf{K}=\mathbf{K C}^{-1} \mathbf{M} \text {. }
$$

This relation has the drawback that it cannot be generalised to a system with singular matrices. However, Caughey and $\mathrm{O}^{\prime}$ Kelly have proven for nonnegative definite systems $\mathbf{K} \geq O, \mathbf{M} \geq 0, \mathbf{C} \geq 0$ that a system has classic eigenmodes if one of these three conditions applies. Another damping type is expressed by Caughey series and is called Caughey's damping.

$$
\mathbf{C}=\mathbf{M} \sum_{\mathbf{j}=0}^{\mathrm{N}-1} \alpha_{i}\left(\mathbf{M}^{-1} \mathbf{K}\right)^{\mathbf{j}}
$$

I this case we express $\mathbf{M}^{-1} \mathbf{C}$ as the power of the product $\mathbf{M}^{-1} \mathbf{K}$. Another damping type is general proportional damping. Here, the coefficients $\alpha_{i}$ at $\mathbf{M}$ and $\mathbf{K}$ are replaced with arbitrary matrix functions $\beta_{i}(\bullet)$. The proportional damping form can be generalised for a positive definite system. If the $1^{\text {st }}$ condition in (52) is multiplied by $\mathbf{M}^{-1}$ and the $2^{\text {nd }}$ condition is multiplied by $\mathbf{K}^{-1}$, then we obtain

$$
\left(\mathbf{M}^{-1} \mathbf{K}\right)\left(\mathbf{M}^{-1} \mathbf{C}\right)=\left(\mathbf{M}^{-1} \mathbf{C}\right)\left(\mathbf{M}^{-1} \mathbf{K}\right), \text { respectively } \mathbf{A B}=\mathbf{B A}
$$




$$
\left(\mathbf{K}^{-1} \mathbf{M}\right)\left(\mathbf{K}^{-1} \mathbf{C}\right)=\left(\mathbf{K}^{-1} \mathbf{C}\right)\left(\mathbf{K}^{-1} \mathbf{M}\right), \text { respectively } \mathbf{A}^{-1} \mathbf{D}=\mathbf{D A}^{-1}
$$

where $\mathbf{A}=\mathbf{M}^{-1} \mathbf{K}, \mathbf{B}=\mathbf{M}^{-1} \mathbf{C}$ and $\mathbf{D}=\mathbf{K}^{-1} \mathbf{C}$. Note that the $3^{\text {rd }}$ condition has not been considered because multiplying by $\mathbf{C}^{-1}$ gives us a similar commutative condition. Eigenvalues, $\mathbf{B}$ and $\mathbf{D}$ are positive because the system is positive definite. It is true that for each two commutative matrices $\mathbf{A}$ and $\mathbf{B}, \beta(\mathbf{A})$ is also commutative from $\mathbf{B}$, where $\beta(x)$ is a smooth real analytic function in the neighbourhood of eigenvalues $\mathbf{A}$. The damping matrix $\mathbf{C}$ is then expressed as the sum

$$
\mathbf{C}=\mathbf{M} \beta_{1}\left(\mathbf{M}^{-1} \mathbf{K}\right)+\mathbf{K} \beta_{2}\left(\mathbf{K}^{-1} \mathbf{M}\right)
$$

Similarly, if we multiply condition 1) by $\mathbf{M}^{-1}$ and 2) by $\mathbf{K}^{-1}$ we obtain

$$
\mathbf{C}=\beta_{3}\left(\mathbf{K M}^{-1}\right) \mathbf{M}+\beta_{4}\left(\mathbf{M K}^{-1}\right) \mathbf{K}
$$

The damping matrices given by equations (56) and (57) guarantees classic eigenmodes. However, due to the special shape of the arguments in functions $\beta(\bullet)$ in (56) and (57), $\mathbf{C}$ does not cover the entire set of real space $R^{N \times N}$, so many damped systems do not have classic eigenmodes. Proportional damping (46) is obtained if we put

$$
\beta_{i}(\bullet)=\alpha_{i} I
$$

The damping matrix expressed by equations (56) and (57) provides a new way of interpreting "Rayleigh's damping" or "proportional damping". In this case the coefficients $\alpha_{i}$ at $\mathbf{M}$ and $\mathbf{K}$ are replaced with arbitrary matrix functions $\beta_{i}(\bullet)$. This damping model is called generalised proportional damping. Functions $\beta_{i}(\bullet)$ are called proportional damping functions that are identical to the damping constants $\left(\alpha_{i}\right)$ in Rayleigh's model. It can be shown that the damping matrix expressed in the form of equations (56) and (57) is the most appropriate representation of Caughey's damping.

\section{Sensitivity analysis}

In recent years, methods have been intensively developed to calculate derivatives of eigenvalues and eigenvectors. This is due to the fact that derivatives of eigenvalues with respect to design variables are important in sensitivity analysis for optimization of mechanical systems [24]. This allows us to modify the design for given situations, for example discrepancies between analyses and experiments when changing design variables, and to indicate changes in the design parameters in order to improve the correlation between analyses and experiments.

Almost all methods for sensitivity analysis use the equation of motion in state space. These methods, however, are disadvantageous because the calculation needs a lot of CPU time and a lot of disk space to save the matrices.

Many sensitivity analysis methods are limited only to systems with symmetric matrices. As we have mentioned, real systems have asymmetric matrices of mass, damping and stiffness, for example the behaviour of structures in the fluid, movement of vehicles on the road, aircraft "flutter", and gyroscopic systems (Fig.1). Sensitivity analysis of these systems cannot be done using the methods for symmetric systems. 


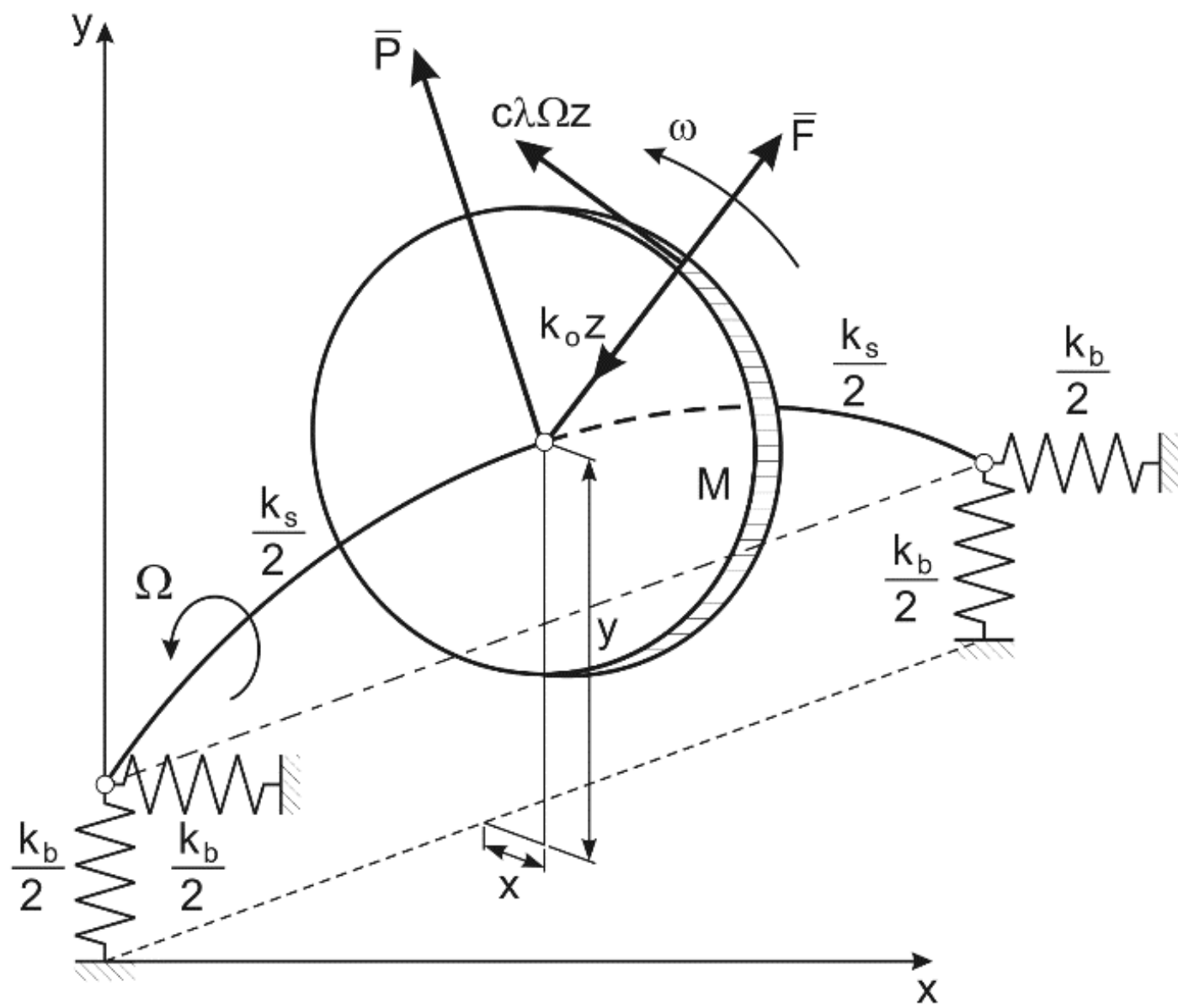

Fig. 1. Gyroscopic system

Therefore in this section we derive an algorithm of the method that does not require a lefthand-side eigenvector. It is based on a quadratic problem in $N$-dimensional space (38)

$$
\left(s^{2} \mathbf{M}^{2}+s \mathbf{C}+\mathbf{K}\right) \mathbf{u}=\mathbf{0}
$$

Differentiating (38) with respect to the design variable $\alpha$ we obtain

$$
\left(s_{j}^{2} \mathbf{M}+s_{j} \mathbf{C}+\mathbf{K}\right) \mathbf{u}_{j, \alpha}=-\left(2 s_{j} \mathbf{M}+\mathbf{C}\right) \mathbf{u}_{j} s_{j, \alpha}-\left(s_{j}^{2} \mathbf{M}_{, \alpha}+s_{j} \mathbf{C}_{, \alpha}+\mathbf{K}_{, \alpha}\right) \mathbf{u}_{j}
$$

where $(.)_{, \alpha}$ represents the derivative (.) with respect to the design variable $\alpha$. Multiplying equation (59) by the vector $\mathbf{u}_{j}^{T}$ we obtain

$$
s_{j, \alpha}=-\mathbf{u}_{j}^{T}\left(s_{j}^{2} \mathbf{M}+s_{j} \mathbf{C}+\mathbf{K}\right) \mathbf{u}_{j, \alpha}-\mathbf{u}_{j}^{T}\left(s_{j}^{2} \mathbf{M}_{, \alpha}+s_{j} \mathbf{C}_{, \alpha}^{T}+\mathbf{K}_{, \alpha}\right) \mathbf{u}_{j}
$$

Transposing equation (60) we obtain

$$
s_{j, \alpha}=-\mathbf{u}_{j, \alpha}^{T}\left(s_{j}^{2} \mathbf{M}^{T}+s_{j} \mathbf{C}^{T}+\mathbf{K}^{T}\right) \mathbf{u}_{j}-\mathbf{u}_{j}^{T}\left(s_{j}^{2} \mathbf{M}_{, \alpha}^{T}+s_{j} C_{, \alpha}^{T}+\mathbf{K}_{, \alpha}\right) \mathbf{u}_{j}
$$

It can be seen from equation (61) that the calculation of eigenvalues derivatives is complex. Therefore, some authors use left-hand-side vectors to calculate eigenvalues derivatives [25]. Another approach is based on the simultaneous calculation of eigenvalues derivatives and eigenvectors [26].

In the following we derive a method where the problems caused by singularity and damping are solved simultaneously from a single algebraic equation. The method calculates 
the derivatives of eigenvalues from the eigenvalue problem and the condition for left-handside vectors.

Transferring the members in equation (59) we obtain

$$
\left(s_{j}^{2} \mathbf{M}+s_{j} \mathbf{C}+\mathbf{K}\right) \mathbf{u}_{j, \alpha}+\left(2 s_{j} \mathbf{M}+\mathbf{C}\right) \mathbf{u}_{j} s_{j, \alpha}=-\left(s_{j}^{2} \mathbf{M}_{, \alpha}+s_{j} \mathbf{C}_{, \alpha}+\mathbf{K}_{, \alpha}\right) \mathbf{u}_{j}
$$

The damped systems eigenvector is normalised in the state space as

$$
\left\{\begin{array}{c}
\mathbf{u}_{j} \\
s_{j} \mathbf{u}_{j}
\end{array}\right\}^{T}\left[\begin{array}{cc}
\mathbf{C} & \mathbf{M} \\
\mathbf{M} & \mathbf{0}
\end{array}\right]\left\{\begin{array}{c}
\mathbf{u}_{j} \\
\boldsymbol{s}_{j} \mathbf{u}_{j}
\end{array}\right\}=\mathbf{u}_{j}^{T}\left(2 s_{, j} \mathbf{M}+\mathbf{C}\right) \mathbf{u}_{j}=1
$$

Differentiating (63) we obtain

$$
\mathbf{u}_{j}^{T}\left(2 s_{j} \mathbf{M}+2 s_{j} \mathbf{M}^{T}+\mathbf{C}+\mathbf{C}^{T}\right) \mathbf{u}_{j, \alpha}+2 \mathbf{u}_{j}^{T} \mathbf{M} \mathbf{u}_{j} s_{j, \alpha}=-\mathbf{u}_{j}^{T}\left(2 s_{j} \mathbf{M}_{, \alpha}+\mathbf{C}_{, \alpha}\right) \mathbf{u}_{j}
$$

Note that unlike the symmetrical systems, equation (64) features transposed coefficient matrices because $\mathbf{M}^{T} \neq \mathbf{M}, \mathbf{C}^{T} \neq \mathbf{C}$ and $\mathbf{K}^{T} \neq \mathbf{K}$. Combining equations (62) and (63) we obtain a single algebraic equation, from which we calculate derivatives of eigenvalues and eigenvectors

$$
\begin{gathered}
{\left[\begin{array}{cc}
s_{j}^{2} \mathbf{M}+s_{j} \mathbf{C}+\mathbf{K} & \left(2 s_{j} \mathbf{M}+\mathbf{C}\right) \mathbf{u}_{j} \\
\mathbf{u}_{j}^{T}\left(2 s_{j} \mathbf{M}+2 s_{j} \mathbf{M}^{T}+\mathbf{C}+\mathbf{C}^{T}\right) & 2 \mathbf{u}_{j}^{T} \mathbf{M} \mathbf{u}_{j}
\end{array}\right]\left\{\begin{array}{c}
\mathbf{u}_{j, \alpha} \\
s_{j, \alpha}
\end{array}\right\}=} \\
=-\left\{\begin{array}{c}
\left(\lambda_{j}^{2} \mathbf{M}_{, \alpha}+s_{j} \mathbf{C}_{, \alpha}+\mathbf{K}_{, \alpha}\right) \mathbf{u}_{j} \\
\mathbf{u}_{j}^{T}\left(2 s_{j} \mathbf{M}_{, \alpha}+\mathbf{C}_{, \alpha}\right) \mathbf{u}_{j}
\end{array}\right\}
\end{gathered}
$$

Numerical solving of sensitivity analysis requires numerical stability of equation (65). This is guaranteed by the matrix $\mathbf{A}^{*}$ non-singularity in equation (65), i.e. $\operatorname{det}\left(\mathbf{A}^{*}\right) \neq 0$. In order to prove that the matrix $\mathbf{A}^{*}$ is non-singular, we introduce the following equations

$$
\mathbf{X}=\left[\begin{array}{ll}
\boldsymbol{\Gamma} & \mathbf{0} \\
\mathbf{0} & \mathbf{1}
\end{array}\right], \mathbf{Y}=\left[\begin{array}{ll}
\boldsymbol{\psi} & \mathbf{0} \\
\mathbf{0} & \mathbf{1}
\end{array}\right]
$$

where $\quad \boldsymbol{\Gamma}=\left[\phi_{1}, \phi_{2}, \ldots ., \phi_{n-1} \mathbf{v}_{j}\right], \quad \boldsymbol{\psi}=\left[\psi_{1}, \psi_{2}, \ldots ., \psi_{n-1} \mathbf{u}_{j}\right], \quad$ and $\quad \mathbf{v}_{j}$ is the left-hand-side eigenvector, and $\mathbf{u}_{j}$ is the right-hand-side eigenvector, which satisfy the following condition

$$
\begin{array}{r}
\left(s_{j}^{2} \mathbf{M}+s_{j} \mathbf{C}+\mathbf{K}\right) \mathbf{u}_{j}=\mathbf{0} \\
\mathbf{v}_{j}^{T}\left(s_{j}^{2} \mathbf{M}+s_{j} \mathbf{C}+\mathbf{K}\right)=\mathbf{0}
\end{array}
$$

Vectors $\phi_{k}$ and $\boldsymbol{\Psi}_{k}$ are any vectors that are independent of $\mathbf{v}_{j}$ and $\mathbf{u}_{j}$. Multiplying the matrix $\mathbf{A}^{*}$ by vectors $\mathbf{X}^{T}$ and $\mathbf{Y}$ we obtain

$$
\begin{aligned}
\mathbf{X}^{T} \mathbf{A}^{*} \mathbf{Y}= & {\left[\begin{array}{ll}
\boldsymbol{\Gamma} & \mathbf{0} \\
\mathbf{0} & \mathbf{1}
\end{array}\right]^{T}\left[\begin{array}{cc}
s_{j}^{2} \mathbf{M}+\lambda_{j} \mathbf{C}+\mathbf{K} & \left(2 s_{j} \mathbf{M}+\mathbf{C}\right) \mathbf{u}_{j} \\
\mathbf{u}_{j}^{T}\left(2 s_{j} \mathbf{M}+2 s_{j} \mathbf{M}^{T}+\mathbf{C}+\mathbf{C}^{T}\right) & 2 \mathbf{u}_{j}^{T} \mathbf{M} \mathbf{u}_{j}
\end{array}\right]\left[\begin{array}{ll}
\boldsymbol{\psi} & \mathbf{0} \\
\mathbf{0} & \mathbf{1}
\end{array}\right]=} \\
& =\left[\begin{array}{cc}
\boldsymbol{\Gamma}^{T}\left(s_{j}^{2} \mathbf{M}+s_{j} \mathbf{C}+\mathbf{K}\right) \boldsymbol{\psi} & \boldsymbol{\Gamma}^{T}\left(2 s_{j} \mathbf{M}+\mathbf{C}\right) \mathbf{u}_{j} \\
\mathbf{u}_{j}^{T}\left(2 s_{j} \mathbf{M}+2 s_{j} \mathbf{M}^{T}+\mathbf{C}+\mathbf{C}^{T}\right) \boldsymbol{\psi} & 2 \mathbf{u}_{j}^{T} \mathbf{M} \mathbf{u}_{j}
\end{array}\right]
\end{aligned}
$$


Since the last columns $\Gamma$ and $\psi$ are the system eigenvectors, we can rewrite equation (68) to the following form

$$
\mathbf{X}^{T} \mathbf{A}^{*} \mathbf{Y}=\left[\begin{array}{ccc}
\tilde{\mathbf{B}} & 0 & \tilde{\mathbf{c}} \\
0 & 0 & \mathbf{c} \\
\mathbf{d}^{T} & 2 & 2 \mathbf{u}_{j}^{T} \mathbf{M} \mathbf{u}_{j}
\end{array}\right]
$$

where $\tilde{\mathbf{B}}$ is the non-singular matrix $(n-1) \times(n-1)$ corresponding to the eigenvalues $\lambda_{j}$, $\mathbf{c}=\mathbf{v}_{j}^{T}\left(2 s_{j} \mathbf{M}+\mathbf{C}\right) \mathbf{u}_{j}, \tilde{\mathbf{c}}$ and $\tilde{\mathbf{d}}$ are non-zero matrices.

Utilising the determinant's property for a partitioned matrix, we can simplify equation (69) to the following form

$$
\operatorname{det}\left(\mathbf{X}^{T} \mathbf{A}^{*} \mathbf{Y}\right)=\operatorname{det}(\tilde{\mathbf{B}}) \times \operatorname{det}\left(\left[\begin{array}{cc}
0 & c \\
2 & 2 \mathbf{u}_{j}^{T} \mathbf{M} \mathbf{u}_{j}
\end{array}\right]-\left[\begin{array}{c}
0 \\
\tilde{\mathbf{d}}^{T}
\end{array}\right][\tilde{\mathbf{B}}]^{-1}\left[\begin{array}{ll}
0 & \tilde{\mathbf{c}}
\end{array}\right]\right)=-2 b \operatorname{det}(\tilde{\mathbf{A}}) \neq 0
$$

We can see that $\operatorname{det}\left(\mathbf{A}^{*}\right) \neq 0$ because matrices $\mathbf{X}$ and $\mathbf{Y}$ are non-singular. In other words, the matrix $\mathbf{A}^{*}$ is non-singular.

\section{Example 2 - Gyroscopic system}

The equation of motion of the gyroscopic system depicted in Fig. 2 has the following form

$$
\mathbf{M u ̈}(t)+(\mathbf{C}+\mathbf{G}) \dot{\mathbf{u}}(t)+(\mathbf{K}+\mathbf{H}) \mathbf{u}(t)=\mathbf{F}(t)
$$

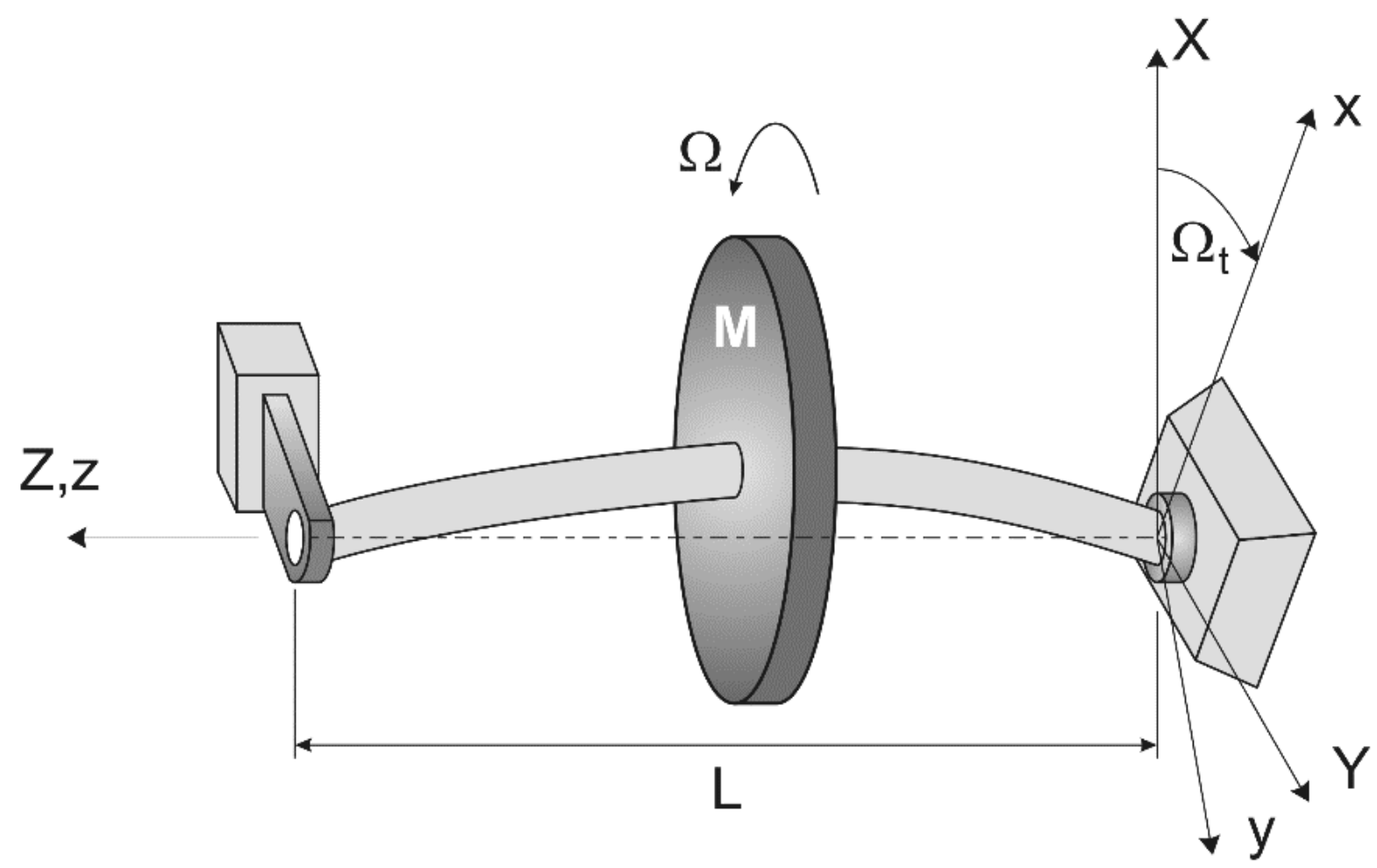

Fig.2 Rotating beam

where $\mathbf{M}, \mathbf{C}, \mathbf{K}$ and $\mathbf{F}$ are matrices of mass, damping, stiffness and external load, $\mathbf{G}$ and $\mathbf{H}$ are the gyroscopic matrix and the centrifugal forces matrix. So the system is asymmetric and the coefficient matrices are as follows 


$$
\begin{gathered}
\mathbf{M}=\left[\begin{array}{cc}
\mathbf{M}_{11} & 0 \\
0 & \mathbf{M}_{22}
\end{array}\right], \quad \mathbf{C}=\left[\begin{array}{cc}
\mathbf{C}_{11} & 0 \\
0 & \mathbf{C}_{22}
\end{array}\right], \quad \mathbf{G}=\left[\begin{array}{cc}
0 & \mathbf{G}_{12} \\
-\mathbf{G}_{12} & 0
\end{array}\right] \\
\mathbf{K}=\left[\begin{array}{cc}
\mathbf{K}_{11} & 0 \\
0 & \mathbf{K}_{22}
\end{array}\right], \quad \mathbf{H}=\left[\begin{array}{cc}
0 & \mathbf{H}_{12} \\
-\mathbf{H}_{12} & 0
\end{array}\right]
\end{gathered}
$$

Matrices $\mathbf{G}$ and $\mathbf{H}$ are given as

$$
\begin{aligned}
& {\left[\mathbf{G}_{12}\right]_{i j}=-2 \Omega\left[\mathbf{M}_{11}\right]_{i j}} \\
& {\left[\mathbf{H}_{12}\right]_{i j}=-h \Omega L \delta_{i j}}
\end{aligned}
$$

Material data are as follows [27]

$$
\begin{aligned}
& \quad m_{0}=1 \mathrm{~kg} / \mathrm{m}, M=1 \mathrm{~kg}, L=1 \mathrm{~m}, K_{1}=K_{2}=L^{2} / 20 \mathrm{Nm}, c=h=1 / 4 \mathrm{Nsm}^{-1}, E I_{x}=4 L^{3} / 5 \pi^{2} \mathrm{Nm}^{2}, \\
& E I_{y}=9 L^{3} / 5 \pi^{2} \mathrm{Nm}^{2}, \Omega=\sqrt{21.6} \pi \mathrm{rads}^{-1} .
\end{aligned}
$$

The number of degrees of freedom is 20 , and the design variable is the beam length $L$. The eigenvalues and their derivatives obtained from (65) by solving in MATLAB are presented in Tab.1, and eigenvector derivatives are shown in Tab.2.

Tab.1 Eigenvalues and their derivatives

\begin{tabular}{|c|c|c|c|c|}
\hline Eigenvalue & \multicolumn{2}{|c|}{ Eigenvalue } & \multicolumn{2}{c|}{ Derivative } \\
\hline & $\operatorname{Re} s_{j}$ & $\operatorname{Im} s_{j}$ & $\operatorname{Re} s_{j, \alpha}$ & $\operatorname{Im} s_{j, \alpha}$ \\
\hline 1 & 2.557 & 0.0 & $2.606 \mathrm{e}+01$ & $-4.859 \mathrm{e}+01$ \\
\hline 2 & -2.813 & 0.0 & $1.885 \mathrm{e}+01$ & $1.700 \mathrm{e}+01$ \\
\hline 3 & $-1.560 \mathrm{e}-01$ & $-8.539 \mathrm{e}+00$ & $6.506 \mathrm{e}+00$ & $4.119 \mathrm{e}+01$ \\
\hline 4 & $-1.560 \mathrm{e}-01$ & $8.539 \mathrm{e}+00$ & $-1.634 \mathrm{e}+01$ & $-3.827 \mathrm{e}+01$ \\
\hline 5 & $1.803 \mathrm{e}-01$ & $-1.232 \mathrm{e}+01$ & $2.086 \mathrm{e}+00$ & $-2.768 \mathrm{e}+00$ \\
\hline 6 & $1.803 \mathrm{e}-01$ & $1.232 \mathrm{e}+01$ & $3.189 \mathrm{e}+00$ & $-1.947 \mathrm{e}+01$ \\
\hline 7 & $-3.427 \mathrm{e}-01$ & $-1.688 \mathrm{e}+01$ & $-1.283 \mathrm{e}-01$ & $2.998 \mathrm{e}+01$ \\
\hline 8 & $-3.427 \mathrm{e}-01$ & $1.688 \mathrm{e}+01$ & $-7.071 \mathrm{e}+01$ & $2.666 \mathrm{e}+01$ \\
\hline 9 & $-3.719 \mathrm{e}-01$ & $-2.944 \mathrm{e}+01$ & $9.281 \mathrm{e}+01$ & $1.581 \mathrm{e}+01$ \\
\hline 10 & $-3.719-01$ & $2.944 \mathrm{e}+01$ & $1.591 \mathrm{e}+01$ & $-2.893 \mathrm{e}+01$ \\
\hline
\end{tabular}


Tab. 2 Derivatives of the $1^{\text {st }}$ eigenvector

\begin{tabular}{|c|c|c|c|c|}
\hline $\begin{array}{c}\text { Degree of } \\
\text { freedom }\end{array}$ & \multicolumn{2}{|c|}{$1^{\text {st }}$ eigenvector } & \multicolumn{2}{c|}{$\begin{array}{c}\text { Derivative of the 1 } 1^{\text {st }} \\
\text { eigenvector }\end{array}$} \\
\hline & $\operatorname{Re} \lambda_{j}$ & $\operatorname{Im} \lambda_{j}$ & $\operatorname{Re} \lambda_{j, \alpha}$ & $\operatorname{Im} \lambda_{j, \alpha}$ \\
\hline 1 & $1.885 \mathrm{e}-02$ & $-1.559 \mathrm{e}-03$ & $8.823 \mathrm{e}-02$ & $5.033 \mathrm{e}-02$ \\
\hline 2 & $-3.304 \mathrm{e}-02$ & $7.835 \mathrm{e}-02$ & $1.012 \mathrm{e}-01$ & $-9.981 \mathrm{e}-01$ \\
\hline 3 & $2.616 \mathrm{e}-04$ & $1.029 \mathrm{e}-02$ & $-7.523 \mathrm{e}-02$ & $6.620 \mathrm{e}-02$ \\
\hline 4 & $4.033 \mathrm{e}-03$ & $-2.394 \mathrm{e}-03$ & $8,9,16 \mathrm{e}-03$ & $8.761 \mathrm{e}-03$ \\
\hline 5 & $-1.400 \mathrm{e}-03$ & $-6.835 \mathrm{e}-04$ & $3.352 \mathrm{e}-04$ & $-7.207 \mathrm{e}-03$ \\
\hline$\cdot$ & $\cdot$ & $\cdot$ & $\cdot$ &. \\
\hline 17 & $-2.3744 \mathrm{e}-05$ & $4.239 \mathrm{e}-04$ & $-8.362 \mathrm{e}-04$ & $1.101 \mathrm{e}-03$ \\
\hline 18 & $5.692 \mathrm{e}-07$ & $1.669 \mathrm{e}-04$ & $6.425 \mathrm{e}-04$ & $3.655 \mathrm{e}-04$ \\
\hline 19 & $1.0351 \mathrm{e}-04$ & $-1.302 \mathrm{e}-04$ & $3.451 \mathrm{e}-04$ & $-1.978 \mathrm{e}-04$ \\
\hline 20 & $-1.474 \mathrm{e}-05$ & $-3.696 \mathrm{e}-05$ & $2.789 \mathrm{e}-04$ & $1.814 \mathrm{e}-04$ \\
\hline
\end{tabular}

\section{Conclusion}

The problem of dynamic analysis of nonconservative linear multiple degrees-of-freedom systems has been considered. Linear structures whose coefficient matrices do not satisfy the classical assumptions of symmetry and definitions arises frequently modern applications. It has been assumed that, in general, the mass, damping, and stiffness matrices are neither symmetric nor positive definite and cannot be simultaneously diagonalized by any linear transformation. Complex eigenvalues of the system are obtained from characteristic equation.

Next, rates of change of eigenvalues and eigenvectors of linear damped discrete systems with respect to the system parameters have been derived. These results are presented in terms of changes in mass, damping, stiffness matrices and complex eigensolutions of the second order system so that the state-space representation of equations of motion can be avoided. These complex eigensolution derivatives can be useful in various application areas, for example, FEM updating, damage detection of structures, design optimization, etc.

Two examples have been used to illustrate the theory herein expounded.

\section{Acknowledgement}

This work was supported by the Slovak Grant Agency VEGA, grant No. 1/0867/18.

\section{REFERENCES}

[1] V. Dekýš, M. Sága, M., Žmindák. Dynamics and Reliability of Mechanical Systems. Scientific Technological Society at University of Žilina, Žilina, 2004 (in Slovak).

[2] F. Trebuňa, M. Hagara. Experimental modal analysis performed by high-speed digital correlation systems. Measurement 2014 (50), 78 - 85.

[3] M. Sivý, M. Musil, Seismic Resistance of Storage Tanks Containing Liquid in Accordance with Principles of Eurocode 8 Standard, Journal of Mechanical Engineering - Strojnícky časopis 2016 (66), No. 2, 79 - 88.

[4] L. Mišík. Functional Analysis. Alfa Bratislava, 1989 (in Slovak).

[5] J. Nagy. Systems of Ordinary Differential Equations. SNTL, Praha (in Czech), 1983. 
[6] C. Kratochvíl, C., M. Nad', L. Houfek. Dynamical Systems: Ordinary Differential Equations. Institute of Termomechanics, Czech Academy of Sciences, Centre of Mechatronics Brno. 2007 (in Czech).

[7] R. Jančo, L. Écsi, P. Elesztős. FSW numerical simulation of aluminium plates by SYSWELD - Part I. Journal of Mechanical Engineering - Strojnicky časopis 2016 (66), No. $1,47-52$.

[8] R. Halama, A. Markopoulos, M. Šofer, Z. Poruba, P. Matušek. Cyclic plastic properties of class C steel emphasizing on ratcheting: Testing and modelling. Journal of Mechanical Engineering - Strojnicky časopis 2015 (65), No. 1, 21 - 26.

[9] J. E. Akin. Finite elements for analysis and design. Academic Press. 1994.

[10] V. Molnár. Computational Fluid Dynamics: Basics with Applications CFD, STU Bratislava, 2006.

[11] T. E. Tezduyar. Finite Element Methods for Fluid Dynamics with Moving Boundaries and Interfaces. In: Encyclopedia of Computational Mechanics, Volume 3: Fluids (eds. E. Stein, R. De Borst and T.J.R. Hughes), John Wiley \& Sons, 2004.

[12] O. C. Zienkiewicz, R. L. Taylor. The Finite Element Method, vol. 1. McGraw-Hill Book Company, Fourth edition, 1991.

[13] V. Kompiš V., M. Žmindák, M. Kaukič. Computer Methods in Mechanics, University of Žilina, Žilina, 2007 (in Slovak).

[14] B. N. Jiang. The Least-Squares Finite Element Method: Theory and Applications in Computational Fluid Dynamics and Electrodynamics, Springer Verlag, 1998.

[15] V. Kompiš, V., M. Toma, M. Žmindák, M. Handrik. Use of Trefftz Functions in NonLinear BEM/FEM. Computers and Structures 2004 (82), 2351 - 2360.

[16] J. Sládek, V. Sládek, L. Jakubovičová. Application of Boundary Element Methods in Fracture Mechanics. Scientific Technological Society at University of Žilina, 2002.

[17] F. Paris, J. Canas, J.. Boundary Element Method. Fundamentals and Applications. Oxford University Press, 1997.

[18] O. Daněk. Condensation of Mathematical Models of Dynamic Systems (in Czech). Journal of Mechanical Engineering - Strojnícky časopis 1997 (48), No. 3, 183 - 190

[19] O. Daněk, J. Kozánek. Mathematical models of dynamics systems of general structure. (in Czech). Journal of Mechanical Engineering - Strojnícky časopis 1998 (49), No. 2, $81-96$.

[20] S. Adhikari. On symmetrizable systems of second kind. Journal of Applied Mechanics 2000 (67), $797-802$.

[21] M. Žmindák, I. Grajciar, J. Nozdrovický. Contribution to modal analyis of nonconservative systems. In: Proceedings of the $3^{\text {rd }}$ International Conference on Dynamics of Civil Engineering and Transport Structures and Wind Engineering. Vrátna, Malá Fatra, 2005 (in Slovak).

[22] S. Adhikari. Modal analysis of linear asymmetric nonconservative systems. Journal of Engineering Mechanics 1999 (125), No. 12, 1372 - 1379.

[23] S. Adhikari. Structural Dynamic Analysis with Generalized Damping Models Identification, Wiley 2014. 
[24] S. Adhikari. Rates of Change of Eigenvalues and Eigenvectors in Damped Dynamic System, AIAA Journal 1999 (37), No. 11, 1452 - 1458.

[25] M. I. Friswell, S. Adhikari. Derivatives of Complex Eigenvectors using Nelson's Method. AIAA Journal 2000 (38), No. 12, 2355 - 2357.

[26] K. M. Choi, H. K. Jo, W.H. Kim, I.W. Lee. Sensitivity analysis of non-conservative eigensystems. Journal of Sound and Vibration 2004 (274), 997 - 1011.

[27] Y.J. Moon, K.M. Choi, H.W. Lim, J.H. Lee, J.H., I.W. Lee. Modified modal methods for calculating eigenpair sensitivity of asymmetric systems. In: The Ninth East Asia Pacific Conference on Structural Engineering and Construction, Vancouver, 2001. 